\title{
DISEÑO DE UN ITINERARIO TURÍSTICO EN TIVISSA A PARTIR DE LA ESTACIÓN METEOROLÓGICA
}

\author{
Òscar Saladié, Jaume Salvat y Salvador Anton \\ Grupo de Investigación de Análisis Territorial y Estudios Turísticos \\ Departamento de Geografía. Universitat Rovira i Virgili
}

\section{RESUMEN}

La existencia de estaciones meteorológicas convencionales, que empezaron su andadura durante la segunda mitad del siglo XIX e inicios del siglo XX, ha hecho posible analizar las variaciones y las tendencias seculares de las diferentes variables meteorológicas a partir de la generación de series regionales. Estas estaciones, que a lo largo de los años han sufrido cambios de emplazamiento, de instrumentos y de observadores, forman parte del patrimonio material de un territorio, pudiéndose convertir en un atractivo turístico. Esta transformación es aún más factible si en los orígenes de la estación se encuentra la figura de un personaje de relevancia histórica, natural de la población donde está localizada la estación o bien una institución que justificó la creación de la estación. En este estudio se exponen los atributos que debería tener una estación para convertirse en un atractivo turístico y se propone un itinerario entorno a la estación meteorológica de Tivissa (Tarragona). Esta estación cuenta con datos desde el año 1911 y sus orígenes están ligados a la figura de Ramon Jardí (Tivissa 1881 - Barcelona 1972), profesor de la Universidad de Barcelona y que, entre otras cosas, diseñó y construyó el prototipo de pluviógrafo de intensidades que lleva su nombre.

Palabras clave: estación meteorológica, itinerario turístico, Tivissa, Dr. Ramon Jardí.

\section{ABSTRACT}

\section{Designing a tourist itinerary in Tivissa by means of the meteorological station}

Analysis of variations and trends of climate during the last 150 years has been possible because instrumental climate data availability recorded in meteorological stations. Changes in location, instruments, exposure and observers have been quite usual along meteorological station history. Meteorological stations have a rich history and they must be considered as material heritage, so they can become a tourism attraction. It is more feasible if there is a famous character or an institution linked with the meteorological station. Here we present the attributes that should have a meteorological station to become a tourist attraction and also showing a proposal of itinerary related to the meteorological station of Tivissa (Tarragona, Spain) with available meteorological records since 1911. It was favoured by Ramon Jardí (Tivissa 1881- Barcelona 1972), professor of the University of Barcelona and designer of Jardí type recording rain intensity gauge and rainfall totaliser.

Key words: meteorological station, tourist itinerary, Tivissa, Dr. Ramon Jardí. 


\section{INTRODUCCIÓN}

Actualmente existe una amplia red de estaciones meteorológicas que proporcionan una ingente cantidad de registros gracias a sensores automáticos, a los cuales hay que añadir aquellos generados desde los satélites meteorológicos. No obstante, la construcción de series regionales de largo recorrido con las cuales poder estimar de una forma robusta la tendencia de la temperatura o la precipitación durante el llamado período instrumental es posible gracias a los datos proporcionados por estaciones convencionales. Estaciones a cargo de personas o colectivos que llevaron y llevan a cabo aún hoy en día la tarea de recogida sistemática de los datos meteorológicos, en la mayoría de los casos voluntaria y desinteresadamente.

Las series meteorológicas temporales pueden tener incorporadas inhomogeneidades generadas por factores no climáticos, relacionados mayoritariamente con cambios de los instrumentos meteorológicos, de su localización o de su entorno (Saladié et al., 2007), pudiendo comprometer la credibilidad de los resultados de los estudios sobre variabilidad climática. Hay que comprobar la homogeneidad de los registros y corregir las inhomogeneidades en caso de existir. Con estos objetivos (detección y corrección) se han desarrollado diferentes metodologías y técnicas (Peterson, et al., 1998). Por todo ello desde la Organización Meteorológica Mundial y en el marco del Programa Mundial del Clima se han dictado las directrices para priorizar la tarea de recopilación de la metadata de las estaciones meteorológicas (Aguilar et al., 2003). El objetivo es dar respuesta a cómo, dónde, cuándo, en qué condiciones y quién obtuvo los datos. Información que debe servir como herramienta auxiliar en la detección de las inhomogeneidades y en la mejora de las correcciones (Saladié, 2011).

Las estaciones meteorológicas con sus instrumentos pueden ser consideradas como patrimonio material de un determinado territorio. La combinación de un patrimonio material, como es la estación meteorológica donde cada instrumento tiene una función y un diseño determinado, con una historia de cambios de instrumentos, localizaciones y observadores (con las potenciales consecuencias de dichos cambios en los registros climáticos) y un personaje o una institución ligados a la población donde se encuentra la estación, pueden convertir dicho patrimonio en un atractivo turístico a través de la creación de un relato asociado a una ruta o a un itinerario.

Los itinerarios tienen un punto de partida y un punto final. La distancia entre estos dos puntos tiene dos componentes esenciales: el recorrido y el tiempo empleado. Es durante el recorrido que se articula un relato que explica una historia. En el caso de los itinerarios esta historia hace referencia a los diferentes elementos patrimoniales, tanto los de carácter físico que se visitan, como a aquellos que son intangibles. Por su parte, el tiempo empleado en desarrollar el relato a lo largo del recorrido determinará en gran parte la profundidad de la experiencia ligada al itinerario. La combinación de estas dos variables, recorrido y tiempo empleado, tiene que garantizar el mantenimiento del interés en el relato por parte del participante durante todo el itinerario.

Los itinerarios o las rutas pueden dar coherencia a una variedad diversa y segregada del patrimonio y ofrecen la oportunidad de articular proyectos de desarrollo territorial basados en su valorización turística (Anton, 1998). El diseño de itinerarios o rutas, entre otros productos, forma parte de una dinámica en la que el componente experiencial juega un papel muy destacado. Experiencias basadas en los sentidos, evocando paisajes y presentando el territorio como un argumento necesario para la experiencia turística (Anton, 2009). La satisfacción de la experiencia turística tendrá una relación directa con el grado de autenticidad de los elementos visitados y la capacidad de participación activa por parte del usuario. Los itinerarios son un buen recurso para dotar la visita turística de un componente formativo y facilitar al mismo tiempo el conocimiento de un entorno más allá incluso del objetivo inicial, facilitando la inclusión de la actividad turística en la estructura productiva de las poblaciones o territorios en los que se inscribe.

Desde un punto de vista histórico, los dos observatorios más importantes de Cataluña son el Observatorio Fabra en Barcelona y el Observatorio del Ebro en Roquetes (Tarragona). El primero se encuentra situado en la vertiente sureste de la sierra de Collserola y fue impulsado desde la Real Academia de Ciencias y Arte de Barcelona. Se trata de un observatorio astronómico, sísmico y meteorológico. Su 
inauguración tuvo lugar el día 7 de abril de 1904, aunque los primeros datos meteorológicos son del año 1913. Por su parte, el Observatorio del Ebro fue fundado por la Compañía de Jesús en las afueras de Roquetes, localidad cercana a Tortosa. Su cometido era el de estudiar las relaciones entre el Sol y la Tierra. La inauguración pública tuvo lugar el día 30 de agosto de 1905 coincidiendo con un eclipse de Sol que fue completo en la zona. Los primeros datos meteorológicos son del año 1905, aunque existen registros de precipitación de una estación situada en las instalaciones que los Jesuitas tenían en la vecina localidad de Jesús desde el año 1880.

En la actualidad, ambos observatorios entre sus actividades ofrecen visitas guiadas. En el caso del Observatorio Fabra las explicaciones se centran en la astronomía, la sismología y la meteorología, mostrándose los dos telescopios centenarios y una sala de estilo modernista. En el Observatorio del Ebro la visita está estructurada entorno a las cuatro secciones de que consta: geomagnetismo, ionosfera, sismología-meteorología y clima-actividad solar.

Algunos otros observatorios catalanes ofrecen visitas guiadas al público en general o a grupos escolares con explicaciones sobre los instrumentos meteorológicos y su funcionamiento, realizándose cursos de meteorología. Entre ellos cabe destacar el Centro Meteorológico de L'Alt Camp en Valls (Tarragona), el Observatorio de Pujalt (Barcelona) o el Observatorio de Sort (Lleida), que cuentan todos ellos con estaciones meteorológicas muy completas.

No obstante, ninguno de los anteriores observatorios está situado en el entramado urbano de los municipios donde se encuentran y no se han producido cambios de localización que permitan hacer un itinerario, sin tener en cuenta los desplazamientos internos por las instalaciones del Observatorio Fabra y del Observatorio del Ebro, dado que la visita queda circunscrita a las instalaciones donde se encuentran los diferentes instrumentos. En el Observatorio Fabra y el Observatorio del Ebro la sección meteorológica no es la que tiene un mayor protagonismo durante la visita. En los casos de Pujalt, Sort y Valls los observatorios tienen un corto recorrido histórico. El de Sort se inauguró en 1985 y el Centro Meteorológico de L'Alt Camp cumplirá 25 años en 2015.

Teniendo como punto de partida la información que aparece en este primer apartado introductorio, el objetivo del presente estudio es analizar si las estaciones meteorológicas pueden convertirse en un atractivo turístico basado en la construcción de un itinerario urbano por los diferentes emplazamientos donde estuvo localizada la estación, asociándolo a la figura de un personaje histórico vinculado con la estación meteorológica.

Para abordar dicho objetivo, en primer lugar se hace una propuesta con aquellas características o atributos que tendrían que cumplir las estaciones meteorológicas para poder convertirse en un atractivo turístico basado en un itinerario. En segundo lugar, se muestra el diseño de un itinerario entorno a la estación meteorológica de Tivissa (Tarragona). Una estación meteorológica creada el año 1911 y que fue auspiciada por Ramon Jardí, natural de Tivissa y que, entre muchas otras cosas, fue el inventor del pluviógrafo de intensidades que lleva su nombre. El Dr. Ramon Jardí ya aparece detectado como recurso referencial de esta localidad en el trabajo realizado por Anton (2000), donde se definen las estrategias para el desarrollo del turismo en el municipio de Tivissa. El estudio se cierra con las conclusiones, los agradecimientos y las referencias bibliográficas.

\section{ATRIBUTOS DE LAS ESTACIONES METEOROLÓGICAS SUSCEPTIBLES DE CONVERTIRSE EN ATRACTIVO TURÍSTICO Y CONSTRUIR UN ITINERARIO}

Todas las estaciones meteorológicas podrían ser consideradas como patrimonio material y en consecuencia convertirse en un atractivo turístico, no obstante, no con todas ellas es posible construir un itinerario basado en un relato histórico y un circuito urbano. A continuación se indican las características o atributos que sería conveniente que cumpliesen dichas estaciones meteorológicas: 
a) Estación con una larga trayectoria histórica.

Las series meteorológicas más antiguas de España se remontan al siglo XVIII. No obstante, no será hasta la segunda mitad del siglo XIX cuando desde el Real Observatorio Astronómico y Meteorológico de Madrid, dirigido por Manuel Rico Sinobas, se gesta una primera red de estaciones y se da impulso a la proliferación de éstas por toda la geografía española. En el caso de Cataluña hay que destacar la labor realizada desde la Sociedad Astronómica de Barcelona, entidad que durante el primer cuarto del siglo XX consiguió establecer una red de casi 200 estaciones pluviométricas (Prohom, 2006), muchas de las cuales posteriormente se completaron con una abrigo meteorológico y los correspondientes termómetros.

Parece lógico afirmar que, con excepciones, cuanto más antigua sea la estación meteorológica más acontecimientos y situaciones cambiantes (localizaciones, observadores, instrumental, entornos, etc.) habrán podido ser documentados. Una información a partir de la cual se podrá diseñar el itinerario y que a su vez nutrirá de contenido las explicaciones realizadas a lo largo del itinerario.

b) Estación que se encuentre en funcionamiento en la actualidad.

El año 1915 la red de la Sociedad Astronómica de Barcelona contaba con un total de 154 estaciones pluviométricas (Febrer, 1930), es decir, estaciones que en la actualidad serían centenarias o casi centenarias. No obstante, muchas de ellas han dejado de existir desde entonces. Teniendo en cuenta que una parte del relato tiene que sustentarse en las características de la estación y de sus instrumentos, es imprescindible que la estación siga estando operativa en la actualidad y de esta manera puedan observarse los diferentes instrumentos que la conforman, comparándolos con otros que hubieran podido existir con anterioridad, ya sea en el mismo emplazamiento o en otro diferente.

c) Estación termo-pluviométrica.

Como tercer atributo, en este estudio proponemos que la estación meteorológica disponga en la actualidad, como mínimo, de pluviómetro y de abrigo meteorológico con los termómetros de máxima y de mínima en su interior. El hecho que se trate de una estación termo-pluviométrica dotará de mucho más contenido las explicaciones relativas a los instrumentos y sus características. Unos instrumentos que tienen una determinada función, que están diseñados para poder recoger o registrar variables meteorológicas de manera precisa y que a lo largo de la historia de la estación han podido ser reemplazados por modelos diferentes.

d) Estación asociada a un personaje histórico o a una institución.

La existencia de un personaje histórico o figura de cierta relevancia ligada a la historia de la estación meteorológica posibilitará la creación de un relato sobre su relación con la estación y que a su vez también tenga en cuenta los hechos más importantes de su biografía, aunque no tengan relación directa con la estación meteorológica. Esta vinculación también podría realizarse en el caso que no existiera un personaje pero sí una institución que promoviera y/o acogiera la estación meteorológica.

e) Emplazamiento adecuado y visitable.

Hay que descartar aquellas estaciones que, estando en la actualidad operativas y cumplir con el resto de características, se encuentren ubicadas en lugares poco accesibles como pueden ser tejados o terrazas. El espacio debe ser suficientemente amplio como para acoger al grupo de personas que realizan el itinerario. A su vez, hay que contar con el permiso del propietario del emplazamiento donde se encuentre la estación, que en la mayoría de casos será el mismo observador. También puede tratarse de una institución u organismo público (p.e. centros educativos) o privado (p.e. instalaciones de una central hidroeléctrica).

\section{PROPUESTA DE ITINERARIO: LA ESTACIÓN METEOROLÓGICA DE TIVISSA Y EL DR. RAMON JARDÍ}

La propuesta que a continuación se presenta consiste en un itinerario guiado que recorre el núcleo urbano de Tivissa siguiendo los lugares relacionados con la estación meteorológica y con su impulsor, el 
Dr. Ramon Jardí, centrándose tanto en dicha figura como en la comprensión de las características de los instrumentos meteorológicos. La selección de esta población de la provincia de Tarragona, que cumple con las características anteriormente indicadas, se ha basado en los trabajos de Saladié et al., (2008) y Saladié (2011). En el primero se analizan las variaciones y la tendencia de la precipitación en el sector nororiental de la Península Ibérica durante el siglo XX a partir de la construcción de una seria regional resultante de combinar un total de 121 estaciones, mayoritariamente situadas en Cataluña. Un análisis en que la metadata de las estaciones juega un papel importante. En el segundo se muestra la historia de las estaciones meteorológicas de la comarca catalana de La Ribera d'Ebre, entre las que se encuentra la estación de Tivissa.

La información para elaborar la historia de los diferentes observatorios analizados se obtuvo mediante la consulta del archivo de la Delegación Territorial en Cataluña de la Agencia Estatal de Meteorología y la de los fondos documentales de la Sociedad Astronómica de Barcelona y del antiguo Servei Meteorològic de Catalunya (ambos custodiados en el archivo del Institut Cartogràfic de Catalunya). También gracias a la información que aparece en el trabajo Memòries Patxot. Atles pluviomètric de Catalunya (Febrer, 1930). Esta tarea se complementó con visitas al emplazamiento de las estaciones, entrevistando al observador y tomando fotografías. Las referencias al Dr. Ramon Jardí están basadas en el trabajo realizado por Saladié y Garcia (2009).

Las vicisitudes acaecidas en la estación meteorológica de Tivissa a lo largo de sus ya más de 100 años de existencia, así como la figura del Dr. Jardí avalan el objetivo de convertirla en un atractivo turístico que complemente la oferta ya existente en esta localidad. Una oferta que aparece relacionada en el estudio llevado a cabo por Anton (2000).

El itinerario propuesto se muestra en la Figura 1. El punto de encuentro es la plaza de la Baranova (a), contigua a la iglesia parroquial. Es el lugar idóneo para introducir el itinerario y contextualizar la situación geográfica de Tivissa que justifica su clima, así como la necesidad de disponer se series de datos meteorológicos para poder caracterizar el clima de una zona. El segundo punto del recorrido es la casa natal del Dr. Ramon Jardí (b). En esta parada del itinerario se aportará información sobre su actividad científica y su relación con Tivissa. Los siguientes puntos del recorrido son los diferentes emplazamientos de la estación meteorológica (c, d, e y f) hasta 1972, relacionando los diferentes observadores y haciendo hincapié en las condiciones del emplazamiento de la estación y de su entorno.

El recorrido termina en las instalaciones donde se encuentra actualmente la estación (g). En esta última parada la explicación estará centrada en las características de dicho emplazamiento en contraposición a la de todos los anteriores, así como en los instrumentos meteorológicos.

Se trata de un itinerario de poco más de $1 \mathrm{~km}$ de distancia entre el punto de salida en la Plaza de la Baranova y la última parada en el emplazamiento de la estación meteorológica. Los contenidos, especialmente los referentes a las características y funciones de los diferentes instrumentos meteorológicos, se adaptarán a las características del grupo participante en el itinerario, con lo cual el tiempo empleado en llevar a cabo la actividad variará entre 60 y 90 minutos.

\subsection{El clima de un territorio: necesidad de datos meteorológicos}

El punto de encuentro e inicio del itinerario es la plaza de la Baranova (Figura 1). Esta plaza se encuentra al lado de la iglesia de Tivissa y antiguamente, como en muchas otras poblaciones, albergó el cementerio. Desde este mirador de más de 40 metros de longitud y 5 metros de alto se contempla, hacia el oeste, un vasto territorio que configura la hoya de Mora con las sierras de Cavalls y La Fatarella en el horizonte, mientras que en dirección norte aparece la imponente silueta de la sierra del Montsant, quedando a sus pies la comarca de El Priorat. 
Figura 1. Propuesta de itinerario turístico por el núcleo urbano de Tivissa.

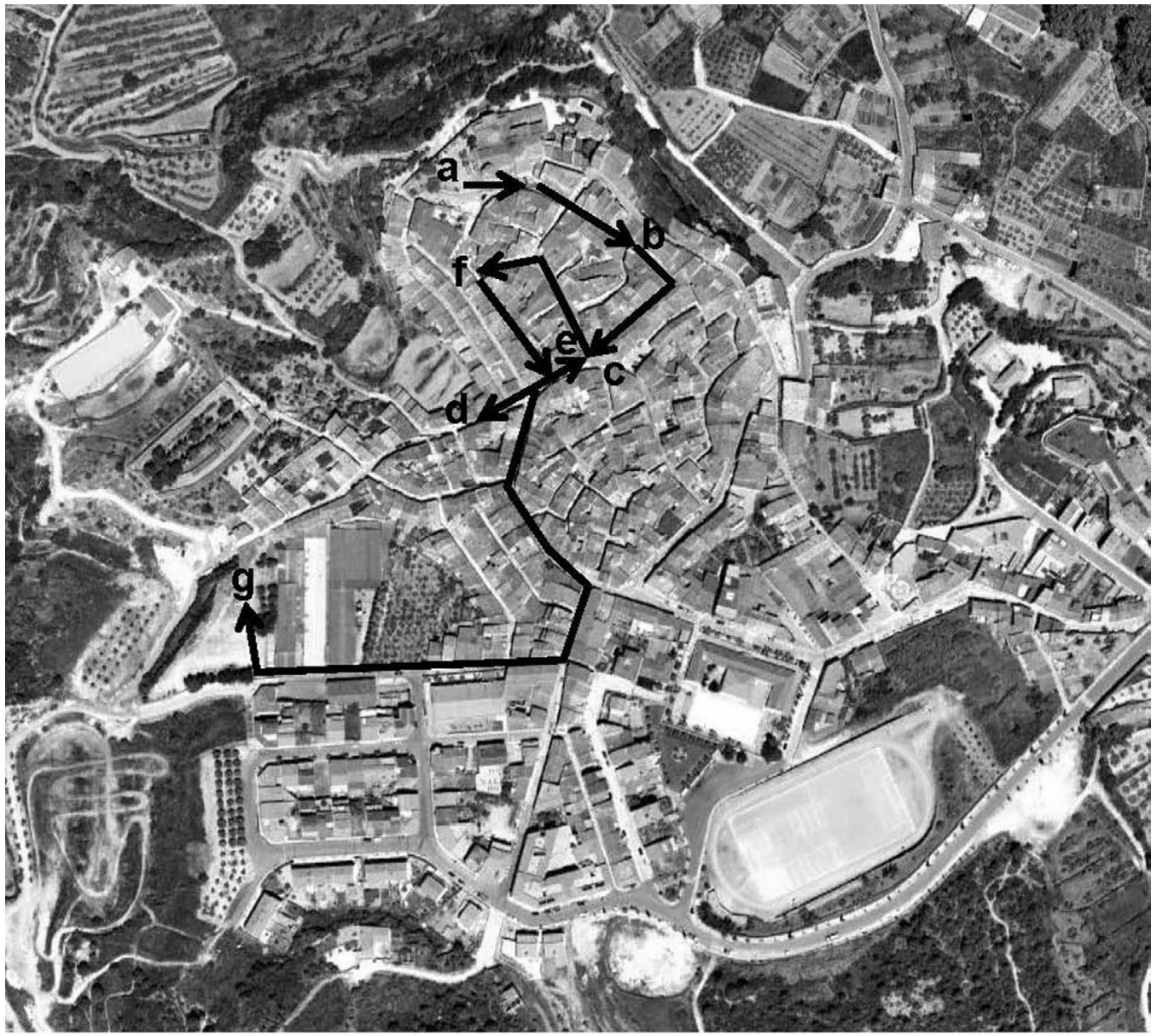

a) Plaza de la Baranova. Punto de encuentro e inicio del itinerario

b) Casa natal del Dr. Ramon Jardí (c. Estanislau Figueras)

c) Residencia de Rosend Loran (c. Castell): 1911-1928

d) Residencia de Víctor Santapau (c. E1 Raval): 1929-1935

e) Ayuntamiento - Jaume Mauri (c. Castell): 1935-1938

f) Cal Vicari - Montserrat y Adolf Brull (c. Abadia): 1938-1972

g) Fábrica Brull - Adolf y Lluís Brull (c. Padrells): 1972-2013

Fuente: Institut Cartogràfic de Catalunya. Elaboración propia.

El visitante que participe en este itinerario turístico deberá aprender que en una misma región climática son la altitud, la distancia al mar y la configuración de la orografía los factores que determinan las diferencias climáticas. Tivissa se encuentra a 310 metros sobre el nivel del mar del cual dista unos $15 \mathrm{~km}$ en línea recta en dirección sureste. No obstante, entre Tivissa y el mar se encuentra la Cordillera Prelitoral Catalana (sierra de Tivissa) que alcaza a superar los 700 metros de altura sobre el nivel del mar. El resultado es que el clima de Tivissa es más suave que el de su entorno más inmediato. En otras palabras, tanto las temperaturas máximas como las mínimas son más moderadas tanto en invierno como en verano. La temperatura media anual del periodo $1971-2000$ en Tivissa es $15,3^{\circ} \mathrm{C}$. Por su parte la temperatura media de las máximas es de $19,9^{\circ} \mathrm{C}$ y la de las mínimas $10,8^{\circ} \mathrm{C}$. Para este mismo período de referencia, el promedio anual de precipitación es de 560,2 $\mathrm{mm}$ de los cuales casi un tercio concentrados en otoño. 
Ahora bien, esta caracterización cuantitativa del clima de Tivissa o de cualquier territorio, sólo es posible si se dispone de una estación meteorológica que proporcione los datos necesarios. Unos datos que en el caso de la estación meteorológica de Tivissa han sido utilizados tanto para estudios de carácter local (Saladié, 1999), como para la construcción de series climáticas regionales (Brunet et. al., 2001; Saladié et al., 2008).

\subsection{Ca Silveri: casa natal del Dr. Ramon Jardí (1881-1972)}

Una vez iniciado el recorrido, la primera parada del itinerario es la casa natal del Dr. Ramon Jardí, ca Silveri, situada en la calle Estanislau Figueras (Figuras 1 y 2a), a poco más de 200 metros de la plaza de la Baranova. Hay que indicar que no está prevista la entrada en dicho edificio y que las explicaciones ser realizarán a pie de calle.

Como se puede observar en la figura 2 (a y b), en la fachada de la casa natal del Dr. Ramon Jardí existe una placa conmemorativa del año 1993, cuando el Ayuntamiento de Tivissa y el Departamento de Física Aplicada de la Universitat Rovira i Virgili organizaron un homenaje. Además de colocar dicha placa también se editó Llibre homenatge al Dr. Jardí (Camps et al., 1993), en el cual participaron tanto el mundo científico y académico, como la gente de Tivissa. En el año 2006 con motivo del $125^{\circ}$ aniversario de su nacimiento tuvieron lugar una serie de actos de homenaje también en Tivissa, organizados por el Ayuntamiento de Tivissa y el Departamento de Geografía de la Universitat Rovira i Virgili. Se ha de destacar una exposición que tuvo por objetivo dar a conocer a los habitantes de Tivissa tanto su figura como su extensa obra.

Fue en esta casa cuando a inicios del siglo XX Ramon Jardí instaló un sismógrafo que había construido él mismo y en 1916 publicó Estudio de las características de un sismógrafo (Jardí, 1916). Hay que recordar que en las proximidades de Tivissa se encuentra el epicentro de un terremoto que tuvo lugar en el año 1845 (Roset, 2009; Font et al., 2010) y que ya fue descrito por el propio Ramon Jardí en un artículo pionero tanto sobre sus características como sobre sus repercusiones (Jardí y Bru, 1921).

Ramon Jardí auspició la creación de la estación meteorológica de Tivissa pero no fue el observador encargado de los registros, puesto que desarrolló su vida profesional y personal en Barcelona, aunque no dejó nunca de pensar en el pueblo que lo vio nacer, como lo demuestra su implicación en diferentes actividades socioculturales llevadas a cabo, así como el hecho de convertirlo en punto de encuentro familiar.

En 1897 inició los estudios superiores en Ciencias Físico-Matemáticas en la Universidad de Barcelona y se licenció en 1902. Un año más tarde obtuvo en Madrid el título de Doctor con una memoria que lleva por título Sobre el movimiento de traslación del Sol (Jardí, 1904) con la cual obtuvo el premio extraordinario. En el año 1904 empezó su carrera docente en la Universidad de Barcelona como Profesor Auxiliar Numerario de la Cátedra de Acústica y Óptica de la Facultad de Ciencias. Finalmente, en el año 1930 se convierte en Catedrático Titular de Acústica y Óptica de la Universidad de Barcelona con una memoria que lleva por título Curvatura de las rayas espectrales dadas por los prismas compuestos (Jardí, 1930).

Ramon Jardí no sólo impartió docencia en la Universidad de Barcelona (Figura 3a), también dio clases en la Escuela Industrial de Barcelona (Figura 3b), concretamente en el Instituto de Electricidad, donde fue profesor de Electricidad y Óptica a partir del año 1917. Con anterioridad, concretamente desde el año 1909, era profesor de Física y Química de la Escuela Municipal de Oficios del Distrito VII de Barcelona.

La actividad científica del Dr. Jardí no quedó circunscrita únicamente a la esfera universitaria, también estuvo vinculado a instituciones como la Real Academia de Ciencias y Artes de Barcelona, la Sociedad Astronómica de Barcelona y la Sección de Ciencias del Institut d'Estudis Catalans. 
Figura 2. Fachada de la casa natal de Ramon Jardí (a), detalle de la placa conmemorativa (b) y pluviógrafo de intensidades Jardí situado en el Observatorio Fabra de Barcelona (c).

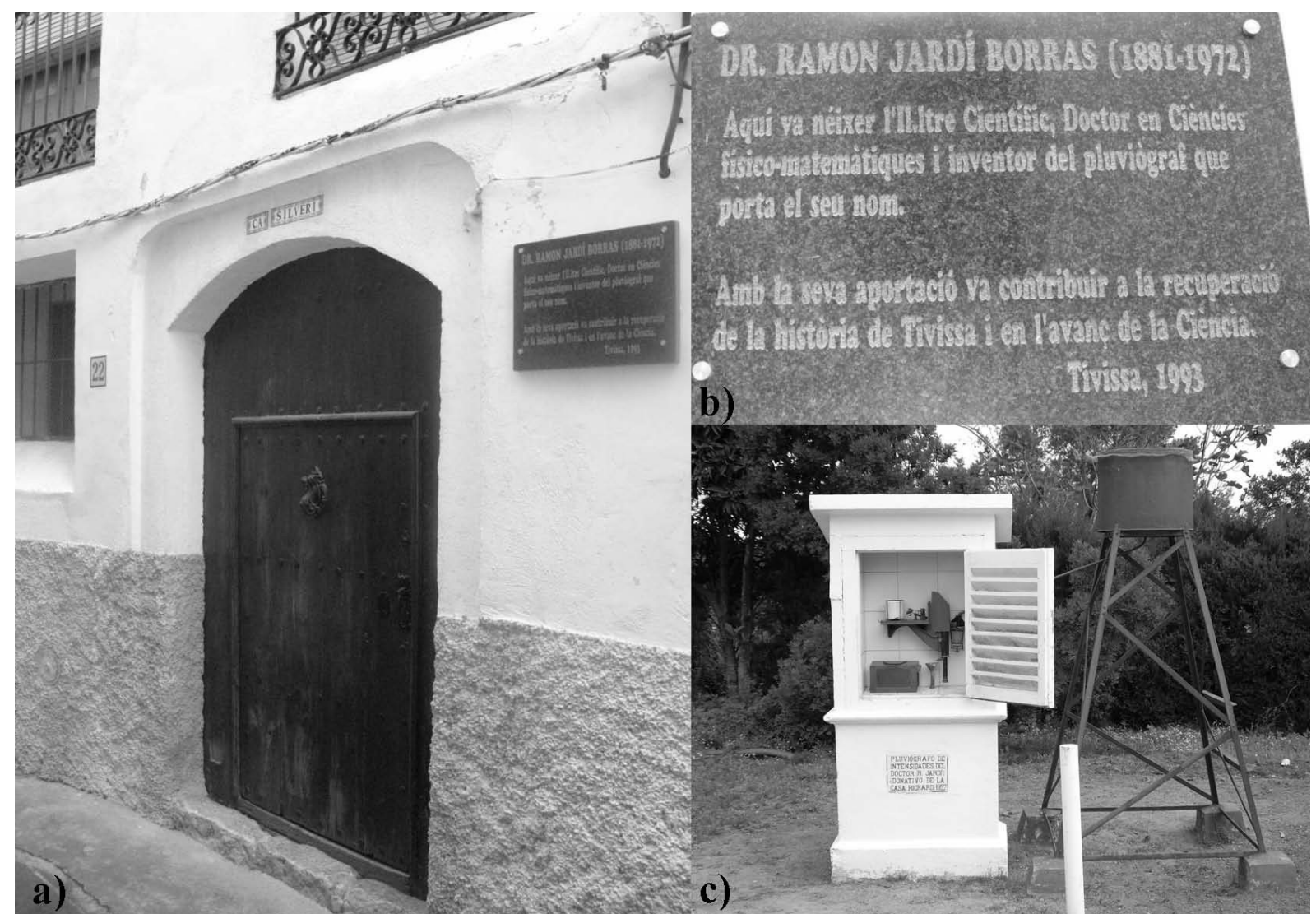

Fotografías: O. Saladié.

Un tercer aspecto a destacar del Dr. Jardí es su papel en dos instituciones clave de la meteorología catalana, como son el Observatorio Fabra de Barcelona y el Servei Meteorològic de Catalunya. Su relación con el Observatorio Fabra se inicia en 1912 cuando Eduard Fontserè es nombrado director de la Sección de Meteorología y Ramon Jardí se convierte en su ayudante, encargándose del buen funcionamiento de los instrumentos meteorológicos, así como también de la reforma de la estación sismológica. Cuando Eduard Fontserè fue nombrado director del Servei Meteorològic de Catalunya en 1921, Ramon Jardí pasó a ser el encargado del laboratorio y de los instrumentos. Ramon Jardí es, o debería ser, conocido y reconocido especialmente por el hecho de haber diseñado y construido un prototipo del pluviógrafo de intensidades que posteriormente llevará su nombre. Su funcionamiento está explicado en la Nota d'Estudi $n^{\circ} 2$ del Servei Meteorològic de Catalunya que lleva por título Un pluviògraf d’intensitats (Jardí, 1921). Un pluviógrafo que posteriormente fue construido en serie por las prestigiosas casas de instrumentos meteorológicos Richard de París y Casella de Londres. El año 1927 la casa Richard hizo donación de dos de estos pluviógrafos. Uno de ellos fue instalado en el Observatorio Fabra de Barcelona, donde aún hoy en día se encuentra (Figura 2c).

Ramon Jardí ejerció de profesor en la Universidad de Barcelona hasta el año 1951 y un año después también dejó de dar clases en la Escuela Industrial, por aquel entonces Escuela de Peritos Industriales de Barcelona. Uno de sus últimos actos públicos tuvo lugar en 1962 durante la inauguración en el Observatorio Fabra de un sismógrafo. Murió en Barcelona el día 5 de junio de 1972 ya cumplidos los 90 años. 
Figura 3. Orla de la Facultad de Ciencias de la Universidad de Barcelona de la promoción 1914-1915 (a) y documento acreditativo como Profesor Titular de la Escuela Industrial de Barcelona del año 1936.
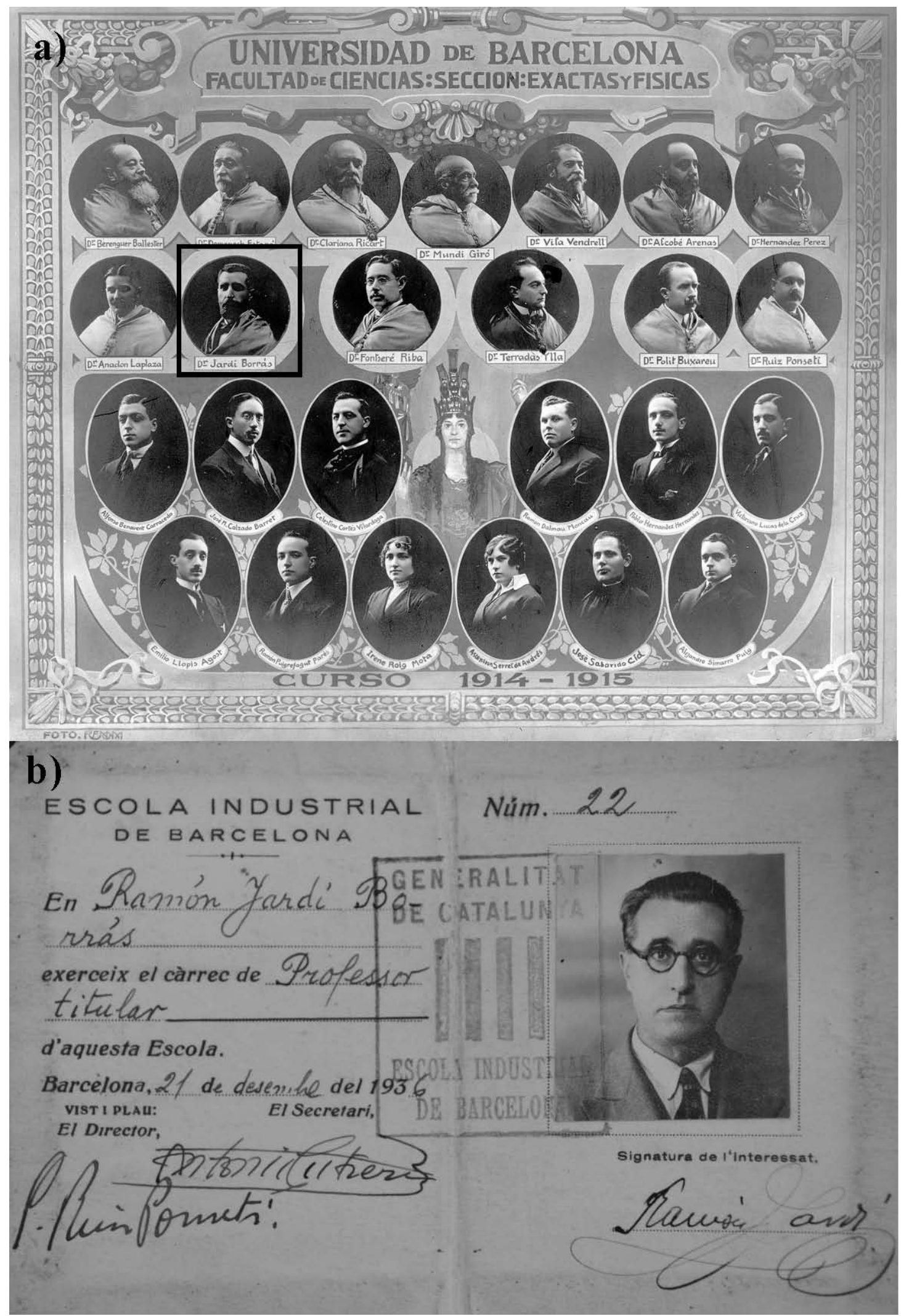

Fuente: archivo particular de la familia de Ramon Jardí Borràs. 


\subsection{La estación meteorológica entre 1911 y 1972: de azotea en azotea}

Una vez contextualizada la vida y la obra del Dr. Ramon Jardí, las siguientes paradas del itinerario sirven para mostrar, también desde la calle, los edificios que acogieron la estación meteorológica entre 1911 y 1972; y a su vez, dar información sobre los diferentes observadores y los instrumentos meteorológicos. Durante aproximadamente los primeros 60 años de existencia de la estación meteorológica de Tivissa los instrumentos estuvieron localizados en azoteas.

Los primeros datos meteorológicos recogidos de una manera sistemática y continua se remontan al mes de octubre del año 1911. En un primer momento se trataba de datos diarios de precipitación registrados en un pluviómetro modelo Hellmann. Formaba parte de la red de estaciones de la Sociedad Astronómica de Barcelona (SAB) y posteriormente del Servei Meteorològic de Catalunya. Ramon Jardí aprovechó los vínculos personales que le proporcionaron sus orígenes para influir en la creación de diferentes estaciones meteorológicas en poblaciones del sur de Cataluña, tal y como hace notar Fontserè (1930:14):

"Desde entonces, la Sociedad creó una sección especial para la pluviometría. Raurich empezó fundando unas cuantas estaciones en El Empordà. Ramon Jardí y Josep Via hicieron una gran cosecha de observatorios en las comarcas del Ebro" (traducción del catalán).

Una de estas estaciones fue la de Tivissa. El primer observador fue el farmacéutico del pueblo, Rosend Loran, encargado de la estación desde 1911 hasta su defunción a finales de 1928. Respecto al emplazamiento en su situación inicial el propio Dr. Jardí en la Nota d’Estudi número 20 del Servei Meteorologic de Catalunya indica (Jardí, 1923: 3):

"Las condiciones de instalación de los instrumentos, sin ser las recomendadas ordinariamente, no son malas, y fue necesario aceptar esta solución, por no ser posible ninguna otra” (traducción del catalán).

A partir del año 1929 el observador es Víctor Santapau, de profesión agricultor. Por aquel entonces junto con el pluviómetro también se disponía de un termómetro de máxima, un termómetro de mínima, un termógrafo y un psicrómetro, así como el abrigo de madera donde estaban los instrumentos (los primeros datos de temperatura son del año 1912). No obstante, con el primer cambio de localización y de observador en 1929 no hubo mejora y los instrumentos también se instalaron en una azotea.

Un nuevo cambio de emplazamiento y de observador tuvo lugar en el año 1935. Desde esa fecha y hasta el año 1938 el encargado pasó a ser Jaume Mauri, el alguacil del pueblo. La estación se situó en el edificio del Ayuntamiento y el sitio escogido fue la azotea de la torre del reloj. A Jaume Mauri le sustituyó Montserrat Brull, la nueva observadora, que había estudiado magisterio. Desde 1938 y hasta 1972 la estación estuvo en la azotea de Cal Vicari (Figura 4a), primero a cargo de Montserrat Brull, y a partir de 1950 a cargo de su hermano Adolf, empresario.

En este punto hay que hacer hincapié en que una vez terminada la Guerra Civil española en 1939, con la derrota republicana, el Servei Meteorològic de Catalunya fue suprimido y las estaciones meteorológicas pasaron a depender del Servicio Meteorológico Nacional, posteriormente Instituto Nacional de Meteorología, convertido desde el año 2008 en Agencia Estatal de Meteorología.

La fotografía del abrigo meteorológico modelo Stevenson (figura 4a) fue tomada a mediados de los años 60 del siglo XX y nos permite comprobar que se trata del mismo abrigo que había sido instalado en la azotea de la casa de Rosend Loran en 1911. Su antigüedad es la razón del pésimo estado de conservación en que se encontraba. Lo corrobora una carta del año 1947 donde Lluís Brull Cedó, tío de Montserrat y Adolf, informa al Jefe del Centro Meteorológico del Ebro, en Zaragoza, que el abrigo continua siendo el del año 1912. Lluís Brull Cedó colaboró con sus sobrinos en las tareas de observador meteorológico. La sustitución del que fuera el primer abrigo meteorológico de la estación de Tivissa no se produjo hasta finales de 1972, coincidiendo con una nueva relocalización, esta vez en las instalaciones de la empresa de la familia Brull.

En la figura 4a también se puede observar que junto al abrigo meteorológico y al pluviómetro se encuentra un pluviógrafo modelo Fuess. Estuvo en funcionamiento hasta el año 1971, cuando fue enviado a Madrid para su reparación. No obstante nunca retornó a Tivissa, a pesar que Adolf Brull envió varias cartas al entonces Jefe del Centro Meteorológico del Ebro, Miguel Liso, reclamándolo sin éxito. 
La distancia entre los diferentes emplazamientos documentados no es muy importante, tal y como se puede observar en la Figura 1. En ningún caso se superan los 150 metros en línea recta.

A finales del año 1972 Adolf Brull trasladó la estación a las instalaciones de la empresa de su propiedad, situada en el extremo suroccidental del entramado urbano de Tivissa (Figura 1). En estas instalaciones se encuentra actualmente, aunque es desde el año 1993 que los instrumentos están localizados en una explanada adyacente a los edificios de la empresa. Actualmente el observador es Lluís Brull Lleó, hijo de Adolf. Así pues, la familia Brull se encarga de la estación meteorológica de Tivissa desde hace más de 70 años. El relevo generacional es bastante frecuente en las estaciones meteorológicas (Saladié, 2011).

\subsection{La estación meteorológica en la actualidad}

La última parada del itinerario se realiza en las instalaciones de la fábrica propiedad de la familia Brull (Figura 1) Los participantes en el itinerario turístico recibirán la información pertinente en relación a los instrumentos meteorológicos (características, funciones, etc.), que será más o menos exhaustiva dependiendo de las características del grupo, y se dará por finalizado el itinerario.

De acuerdo con las recomendaciones de la Organización Meteorológica Mundial las estaciones deberían estar en un lugar llano y libre de obstáculos. Es decir, sin árboles, edificios ni otras construcciones en las proximidades. También sería deseable prever que las modificaciones futuras de las condiciones del entorno sean mínimas, puesto que, en caso contrario, pueden introducir sesgos artificiales en los registros climáticos.

La estación meteorológica de Tivissa es termo-pluviométrica (Figura 4b) y, tal y como ya hemos indicado, pertenece a la red de la Agencia Estatal de Meteorología con el código 9971. Dispone de un pluviómetro modelo Hellmann desde sus inicios. La Sociedad Astronómica de Barcelona generalizó este modelo de pluviómetro en Cataluña. Consiste en dos vasos longitudinales acoplados. El agua entra en el pluviómetro por el primer vaso que acaba en forma de embudo y con una inclinación para evitar la pérdida de gotas de agua por salpicaduras. El agua es recogida en una vasija que se encuentra en su interior desde donde posteriormente se vierte en una probeta graduada para medir la cantidad de lluvia caída. La boca circular de este modelo de pluviómetro tiene un área de $200 \mathrm{~cm}^{2}$ y un diámetro de 159,6 $\mathrm{mm}$. No obstante algunas estaciones que ya existían con anterioridad a la creación de la red de la Sociedad Astronómica de Barcelona disponían de pluviómetros con una boca de $400 \mathrm{~cm}^{2}$ de área y de $226 \mathrm{~mm}$ de diámetro (Fontserè, 1923). Se trata de modelos de pluviómetro Tonnelot y Richard (Observatorio Central Meteorológico, 1915). Entre las estaciones catalanas con pluviómetros de tales características se encontraban Vilafranca del Penedès (Barcelona) y el Observatorio del Ebro en Roquetes (Tarragona).

Un aspecto que ha cambiado en el pluviómetro modelo Hellmann es el material. Los primeros eran de chapa galvanizada, es decir, recubiertos por una capa de zinc con el objetivo de protegerlo de la corrosión y eran de color gris. Posteriormente se hicieron pruebas barnizándolo o pintándolo de blanco con el objetivo de minimizar las pérdidas del agua de su interior debido a evaporación o por grietas en las soldaduras (Sevruk, 1974a, b). En la actualidad también se encuentran de otros materiales, como latón, acero inoxidable o policarbonato.

De acuerdo con Fontserè (1923) el pluviómetro debería estar, como mínimo, a 5 metros de distancia de cualquier obstáculo y la parte más elevada de dicho obstáculo, si lo hubiera, no debería exceder $45^{\circ}$ respecto al plano de la boca del pluviómetro.

Para medir las temperaturas, la estación meteorológica de Tivissa cuenta con un termómetro de máxima, un termómetro de mínima, un termómetro para medir la temperatura a las $8 \mathrm{~h}$ de la mañana y un termógrafo. Los mismos instrumentos disponibles en 1912, a los que había que añadir un psicrómetro (Jardí, 1923). Todos ellos han estado siempre dentro de un abrigo meteorológico que tiene por objetivo proteger a los instrumentos del viento, así como evitar la incidencia directa e indirecta de la radiación solar. 
Figura 4. Localización de la estación meteorológica a mediados de los años 60 del siglo XX (a) y la estación en la actualidad (b).
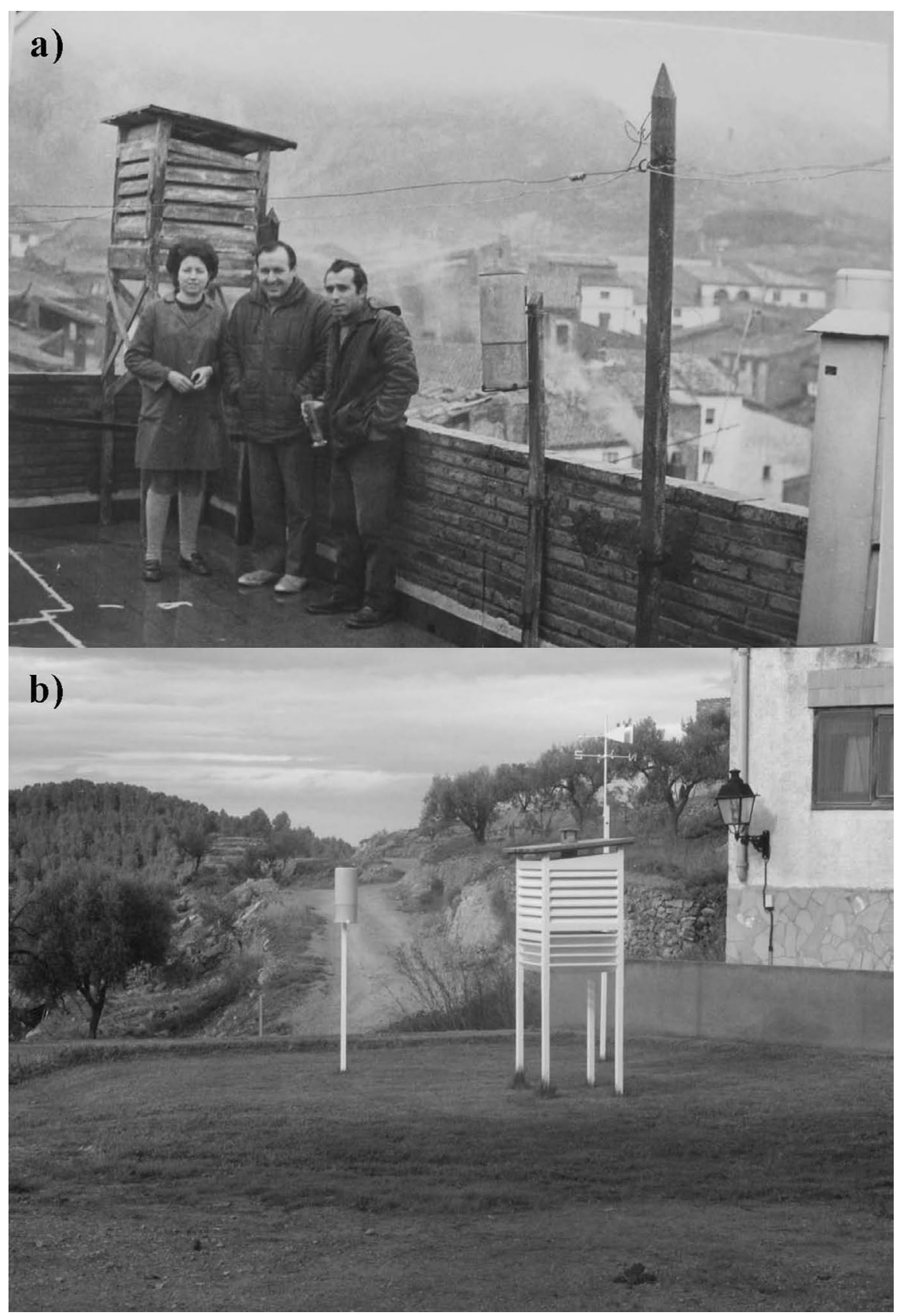

Fotografías: AEMET (a) y O. Saladié (b)

La tipología de abrigos meteorológicos y otro tipo de protectores de los termómetros es amplia y variada. Durante el siglo XIX eran descritos por los coetáneos como simples atriles, facistoles o endebles techados (Brunet et al., 2004). Durante la segunda mitad del siglo XIX se generaliza un nuevo abrigo, el Montsouris, que ofrece una mayor protección a los instrumentos aunque está abierto por la parte frontal y no dispone de base. Entre los observatorios que dispusieron de dicho abrigo meteorológico se encuentra, entre otros, el ya mencionado de Vilafranca del Penedès (Baltà, 1890).

La estación meteorológica de Tivissa ha dispuesto del mismo modelo de abrigo meteorológico a lo largo de los últimos 100 años. Un abrigo que Jardí (1923:3) describe como:

"Modelo recomendado por el Observatorio Central Meteorológico" (traducción del catalán). 
En España es conocido como modelo Galbis (Fontserè, 1923), apellido del que fuera director del Observatorio Central Meteorológico. A nivel internacional se corresponde con el abrigo meteorológico modelo Stevenson.

Las características de dicho abrigo están excelentemente descritas por Fontserè (1923:25-27):

"El sistema de persianas es doble. Las exteriores están inclinadas hacia fuera e interceptan los rayos del Sol incluso en el horizonte y las interiores, así como las que forman una pequeña falda por debajo la caja, están inclinadas hacia dentro e interceptan la irradiación directa de la tierra y de los objetos expuestos al Sol. La puerta del abrigo, que también es de persianas, tiene las bisagras en la parte inferior y durante la observación se la deja colgando. La cubierta está formada de un doble techado de madera [...]. El piso del cajón está constituido por un doble juego de listones, que deja pasar el aire, pero no la reverberación del terreno. Dentro del cajón, separados de las paredes, hay algunos listones traveseros que sirven para sostener los termómetros, donde han de estar fijados con el depósito en el aire y sin tocar la madera ni ningún otro objeto. El conjunto del abrigo está pintado de blanco [...]. El piso del cajón ha de estar situado a la altura del pecho del observador [...] y aún es preferible situarlo unos $35 \mathrm{~cm}$ más arriba y servirse, para la observación, de un taburete o de una pequeña escalera de madera. La orientación del abrigo ha de ser con la puerta exactamente hacia el Norte, para que al abrirla no penetren los rayos del Sol hasta los termómetros [...]. Al pie del abrigo, y en un buen trozo a su alrededor, el suelo ha de estar cubierto de hierba, precaución que disminuye mucho la irradiación" (traducción del catalán).

La cita es ciertamente muy larga, pero es difícil superar esta descripción del Dr. Eduard Fontserè de hace 90 años.

Atendiendo a las características de los abrigos meteorológicos modelos Montsouris y Stevenson, no es de extrañar que las temperaturas máximas registradas en los termómetros situados en el primero sean más elevadas que en la de los mismos termómetros situados en el interior del segundo, mientras que ocurre todo lo contrario con las temperaturas mínimas (Brunet et al., 2004, 2010) y, por consiguiente, se incorpora un sesgo en los registros de temperatura en aquellos observatorios que contaron en sus orígenes con un abrigo Montsouris y que fue posteriormente sustituido por uno modelo Stevenson.

Como se puede observar en la figura 4b, el tejado del abrigo meteorológico de la estación de Tivissa en la actualidad es de chapa, hace pendiente para evitar la acumulación de agua e incorpora una chimenea para favorecer el movimiento del aire en el interior del abrigo.

\section{CONCLUSIONES}

Las estaciones meteorológicas generan una ingente cantidad de datos que posibilitan caracterizar el clima de una zona y a su vez, en el caso de aquellas con una larga trayectoria histórica, también permiten la creación de series regionales con las cuales establecer la deriva térmica o pluviométrica de un determinado territorio, es decir las variaciones y la tendencia de las diferentes variables meteorológicas.

El interés por conocer y comprender las características de los instrumentos meteorológicos, así como la de los emplazamientos donde están ubicados, no tiene por que quedar circunscrito a determinados segmentos de la población vinculados con la meteorología y la climatología. Las estaciones meteorológicas son por ellas mismas un atractivo turístico con un fuerte componente pedagógico. No obstante, este interés aún puede ser mayor y hacerse extensivo a un mayor número de población si las explicaciones sobre las características de los instrumentos meteorológicos se asocian a un itinerario urbano que cubra las diferentes localizaciones en que la estación haya estado ubicada. Más todavía si en el relato que da contenido al itinerario también aparece la figura de un personaje histórico de cierta relevancia, natural o vinculado a la población y que hubiera tenido relación con la estación meteorológica.

En este estudio han sido definidos las características que tendría que cumplir una estación meteorológica para convertirse en un atractivo turístico basado en un itinerario y se hace una propuesta entorno a la estación de la localidad tarraconense de Tivissa. Una población que cuenta con datos meteorológicos desde el año 1911 registrados en una estación que ha sufrido diferentes cambios de localización y que en sus orígenes estuvo vinculada al Dr. Ramon Jardí (1881-1972), profesor de la Universidad de Barcelona, inventor del pluviógrafo de intensidades que lleva su nombre y natural de Tivissa. 
La propuesta de itinerario, con un recorrido a pie de $1 \mathrm{~km}$, cuenta con un total de 6 paradas sin tener en cuenta el punto de encuentro en la plaza contigua a la iglesia parroquial y que sirve para introducir y contextualizar la actividad. Las que cuentan con mayor contenido son la situada enfrente la casa natal del Dr. Jardí y la última en el emplazamiento donde se encuentra actualmente la estación meteorológica.

Los contenidos relacionados con la figura de Ramon Jardí y con las características y funcionamiento de los diferentes instrumentos de la estación meteorológicas se adaptaran a las características del grupo que realice la actividad, hecho que influirá en el tiempo necesario para recorrer el itinerario y puede variar entre 60 y 90 minutos.

Finalmente, un itinerario de estas características puede ofrecer la oportunidad de articular proyectos que tengan en cuenta otros atractivos o recursos turísticos existentes ya sea en el propio núcleo urbano (entre otros, la iglesia parroquial, diferentes casas señoriales o los portales de la antigua muralla medieval), o bien en sus inmediaciones (el poblado ibérico del Castellet de Banyoles en Tivissa, o las pinturas rupestres de La Font de Vilella).

\section{AGRADECIMIENTOS}

Este estudio ha sido realizado en el marco del proyecto "Turismo, movilidad residencial y competitividad territorial. Respuestas locales a las dinámicas de cambio global” (GLOBALTUR, CSO2011-23004), financiado por el Ministerio de Ciencia e Innovación.

Los autores desean agradecer a dos evaluadores anónimos sus comentarios y sugerencias.

\section{BIBLIOGRAFÍA}

AGUILAR, E., AUER, I., BRUNET, M., PETERSON, T. C. y WIERINGA, J. (2003): Guidelines on climate metadata and homogenization. WMO-TD 1186. Organización Meteorológica Mundial. Ginebra.

ANTON, S. (1998): "Rutas e itinerarios turísticos culturales. Propuestas y desafíos", KARIS (Observatorio Europeo de Políticas Culturales, Urbanas y Territoriales), 8, pp. 11-18.

ANTON, S. (2009): "El consum d'experiències i emocions. Perspectives per a la innovació en turisme i oci a Catalunya", Paradigmes, 3, pp. 93-100.

ANTON, S. (Dir.) (2000): Estratègies per al desenvolupament del turisme al municipi de Tivissa, Patronat de Turisme de la Diputació de Tarragona - SAM, Tivissa.

BALTÀ, J. (1890): Estación meteorológica de Vilafranca del Panadés. Observaciones efectuadas en dicha estación durante el año 1889. Establecimiento Tipográfico Pedro Alegret y Vilaró. Vilafranca del Penedès.

BRUNET, M., AGUILAR, E., SALADIÉ, O., SIGRÓ, J. y LÓPEZ, D. (2001): "The variations and trends of the surface aire temperature in Northeastern of Spain from middle nineteenth century onwards", en M. BRUNET y D. LÓPEZ (Eds.): Detecting and modelling regional climate change and associated impacts. Springer. Berlín-Heidelberg-Nueva York, pp. 81-93.

BRUNET, M., ASÍN, J., SIGRÓ, J., BAÑón, M., GARCíA, F., AGUILAR, E., PALENZUEla, J.E., PETERSON, T.C. y JONES, P.D. (2010): "The minimization of the screen bias from ancient western mediterranean air temperatura records: an exploratory statistical analysis", International Journal of Climatology, DOI:10.1002/joc2192.

BRUNET, M., BAÑÓN, M., GARCÍA, F, AGUILAR, E., SAlADIÉ, O., SIGRÓ, J., ASÍN, J. y LÓPEZ, D. (2004): "Una aproximación experimental tendente a la minimización del sesgo artificial asociado al tipo de garita meteorológica a través de la observación dual de la temperatura del aire", en La meteorología y el clima atlánticos. Publicaciones de la Asociación Meteorológica Española. Badajoz, pp. 91-103.

CAMPS, J., ESCODA, J. y MASSONS, J. (Eds.) (1993): Llibre homenatge al Dr. Jardí. Ajuntament de Tivissa. Tivissa.

FEBRER, J. (1930): Memòries Patxot. Atles pluviomètric de Catalunya. Institució Patxot. Barcelona. 
FONT, E., MARTÍNEZ-SOLARES, J.M., MASANA, E. y SANTANCH, P. (2010): "La serie sísmica de Tivissa, 1845 (Cadenas Costeras Catalanas): los deslizamientos del barranco del Manou", Revista de la Sociedad Geológica de España, n 23, pp. 69-80.

FONTSERÈ, E. (1923): Instruccions meteorològiques per als observadors rurals. Editorial Políglota. Col. Minerva número XL. Barcelona.

FONTSERE, E. (1930): "Pròleg", en J. FEBRER: Memòries Patxot. Atles pluviomètric de Catalunya. Institució Patxot. Barcelona, pp. 9-16.

JARDÍ, R. (1904): Sobre el movimiento de traslación del Sol. Memoria presentada para aspirar al título de Doctor en Ciencias Físico-Matemáticas. Tipolitografía de José Casamajó. Barcelona.

JARDÍ, R. (1916): Estudio de las características de un sismógrafo. Real Academia de Ciencias y Artes de Barcelona. Barcelona.

JARDÍ, R. (1921): Un pluviògraf d'intensitats. Nota d'Estudi núm. 2 del Servei Meteorològic de Catalunya. Barcelona.

JARDÍ, R. (1923): Deu anys d'observacions termopluviomètriques a Tivissa. Nota d'Estudi núm. 20 del Servei Meteorològic de Catalunya. Barcelona.

JARDÍ, R. (1930): Curvatura de las rayas espectrales dadas por los prismas compuestos. Memoria presentada para tomar parte en las oposiciones a la cátedra de Acústica y Óptica vacante en la Univesidad de Barcelona. Barcelona.

JARDÍ, R. y BRU, M. (1921): “Terremoto catalán de 1845”, Ibérica, no 361-362, pp. 60-62.

OBSERVATORIO CENTRAL METEOROLÓGICO (1915): Resumen de las observaciones meteorológicas efectuadas en la Península Ibércia y algunas de sus islas adyacentes. Observatorio Central Meteorológico. Madrid.

PETERSON, T. C., EASTERling, D. R., KARL, T. R., GROISMAN, P., NiCHOLS, N., PLUMMER, N., TOROK, S., AUER, I., BÖHM, R., GUllET, D., VINCENT, L., HEINO, R., TUOMENVIRTA, H., MESTRE, O., SZENTIMREY, T., SAlingER, J., FORLAND, E. J., HANSSEN-BAUER, I., ALEXANDERSSON, A., JONES, P. D. y PARKER, D. (1998): "Homogeneity adjustments of in situ atmospheric climate data: a review", International Journal of Climatology, $\mathrm{n}^{\circ}$ 18, pp. 1493-1517.

PROHOM, M. (2006): "La contribución de la Sociedad Astronómica de Barcelona en la difusión de las observaciones meteorológicas en Cataluña (1910-1923)”, Investigaciones Geográficas, n 40, pp. 141-155.

ROSET, J. (2009): "El terratrèmol de Tivissa de l'any 1845", Miscel-lània del Centre d'Estudis de la Ribera d'Ebre, no 19 , pp. 255-264.

SALADIÉ, O. (1999): "Canvis en la dinàmica de les precipitacions: Tivissa 1912-1997", Miscel-lània del Centre d'Estudis de la Ribera d'Ebre, n' 13, pp. 69-94.

SALADIÉ, O. (2011): "Cien años de observaciones meteorológicas en la comarca catalana de Ribera d'Ebre (1911-2011)", Cuadernos Geográficos, no 48, pp. 109-133.

SALADIÉ, O. y GARCIA, M. (2009): "Ramon Jardí: un tivissà a l’Observatori Fabra i al Servei Meteorològic de Catalunya", Miscel-lània CERE, 19, pp. 219-230.

SALADIÉ, O., BRUNET, M., AGUILAR, E., SIGRÓ, J. y LÓPEZ, D. (2007): Observar la lluvia en Cataluña: 150 años de registros. Publicacions de la URV. Tarragona.

SALADIÉ, O., BRUNET, M., AGUILAR, E., SIGRÓ, J. y LÓPEZ, D. (2008): "Variacions i tendència de la precipitació al sector nord-oriental de la península Ibèrica durant el segle XX", Revista de Geografia, $\mathrm{n}^{\mathrm{o}} 5$, pp. 25-46.

SEVRUK, B. (1974a): "Evaporation losses from containers of Hellmann precipitation gauges", Hydrological Sciences Bulletin, $\mathrm{n}^{\circ} 19$, pp. 231-236.

SEVRUK, B. (1974b): "Correction for the wetting loss of a Hellmann precipitation gauge", Hydrological Sciences Bulletin, $\mathrm{n}^{\circ} 19$, pp. 549-559. 